\title{
Forecasting Air Passenger Traffic by Support Vector Machines with Ensemble Empirical Mode Decomposition and Slope-Based Method
}

\author{
Yukun Bao, Tao Xiong, and Zhongyi Hu \\ Department of Management Science and Information System, School of Management, \\ Huazhong University of Science and Technology, Wuhan 430074, China \\ Correspondence should be addressed to Yukun Bao, yukunbao@mail.hust.edu.cn
}

Received 28 August 2012; Accepted 3 October 2012

Academic Editor: Carlo Piccardi

Copyright (C) 2012 Yukun Bao et al. This is an open access article distributed under the Creative Commons Attribution License, which permits unrestricted use, distribution, and reproduction in any medium, provided the original work is properly cited.

\begin{abstract}
With regard to the nonlinearity and irregularity along with implicit seasonality and trend in the context of air passenger traffic forecasting, this study proposes an ensemble empirical mode decomposition (EEMD) based support vector machines (SVMs) modeling framework incorporating a slope-based method to restrain the end effect issue occurring during the shifting process of EEMD, which is abbreviated as EEMD-Slope-SVMs. Real monthly air passenger traffic series including six selected airlines in USA and UK were collected to test the effectiveness of the proposed approach. Empirical results demonstrate that the proposed decomposition and ensemble modeling framework outperform the selected counterparts such as single SVMs (straightforward application of SVMs), Holt-Winters, and ARIMA in terms of RMSE, MAPE, GMRAE, and DS. Additional evidence is also shown to highlight the improved performance while compared with EEMD-SVM model not restraining the end effect.
\end{abstract}

\section{Introduction}

Air passenger traffic forecast is of great importance for airlines and civil aviation authorities. For airlines, accurate forecasts play an increasingly important role in the revenue management. It helps to reduce the airlines' risk by objectively evaluating the demand of the air transportation business [1]. For civil aviation authorities, air passenger traffic forecast provides a concrete basis for planning decisions in air transport infrastructure. For example, the Civil Aviation Authority (CAA) in UK has responsibility for regulating the air transport industry in the UK and advising the government's Department of the Environment, Transport and the Regions (DETR) on air transport matters [2]. DETR was to present the national forecasts periodically for the future demand for air travel, by passenger numbers, at UK airports 
as a whole since the 1980s. The last published report was DETR [3]. Later on, the Department for Transport (DFT) continued to publish several reports with regard to air passenger demand forecast [4-6]. As the air passenger traffic series are typically considered a nonlinear and nonstationary time series with seasonality, forecasting air passenger traffic remains challenging.

In the past decades, academic researchers and practitioners have made many contributions to air passenger traffic forecast. Most of the quantitative forecasting models abounded in the literature can fall into two categories, namely, econometric modeling and time series. In the econometric modeling area, pioneering works can be found in [1,7]. Most econometric models aimed to reveal the relationship between air passenger traffic flow and selected economic or social supply variables such as geoeconomic and service-related factors.

Compared with econometric modeling, little attention has been paid on time series models in air passenger traffic forecast. The important research work was done by Grubb and Mason [2]. They examined the application of damped trend Holt-Winters method with UK air passengers' data on long lead time forecast and indicated that univariate forecasting may have some advantages over multivariate econometric modeling for long lead times. A univariate forecast depends only on the past of the series and not on estimated relationships between the series and exogenous variables, and it does not require forecasts of the exogenous variables, which will themselves be subject to uncertainty. The same research effort on air passenger traffic forecast by time series methods can be found in the literature [8]. Due to the complexity of econometric modeling in variables selection and testing, time series approach is a promising alternative in air passenger traffic forecast though they are handicapped by their inability to indentify the causes of air passenger traffic growth with clear interpretation.

Usually, the above time series models can provide good forecasts when the air passenger traffic series under study is linear or near linear with explicit seasonality and trend. However, in real work air passenger traffic series, there is a great deal of nonlinearity and irregularity along with implicit seasonality and trend. Poor performance can be found frequently in using the traditional time series methods in practice. The main reason is that the underlying assumption of these traditional time series methods is linearity and they cannot capture the nonlinear patterns hidden and recognize the irregularity well. Recent research efforts on modeling time series with complex nonlinearity, dynamic variation, and high irregularity provided two promising directions. One is to establish emerging artificial intelligence models such as artificial neural networks (ANNs), support vector machines (SVMs), and genetic programming (GP). The earlier literature on air passenger traffic forecast by ANN can be found in $[9,10]$. The other is to integrate data decomposition techniques such as empirical mode decomposition (EMD) or ensemble empirical mode decomposition (EEMD, an updated version of EMD) into an unified modeling framework to forecast complex nonlinear time series with great fluctuation and irregularity. Such research effort could be seen in [11-13] and so-called as decomposition-ensemble modeling framework.

Under the principle of decomposition-ensemble modeling framework, this study proposes an ensemble empirical mode decomposition (EEMD) based support vector machines (SVMs) modeling approach for air passenger traffic forecast. Specially, the end effect issue ignored in [11-13] is addressed and the corresponding slope-based method proposed by Dätig and Schlurmann [14] is incorporated into decomposition and ensemble modeling framework to restrain the end effect occurred during the shifting process of EEMD technique. In this proposed methodology, the original air passenger traffic series were first decomposed into a finite and often a small number of intrinsic mode functions (IMFs) and a residual by applying EEMD [15] with slope-based method. After the components 
(IMFS and a residual) were adaptively extracted via EEMD, each component was modeled by an independent SVM (in formulation of support vector regression, SVR) model to forecast the component series, respectively. Finally, the forecasts of all components were aggregated using another independent SVM model, which models the relationship among the IMFs and the residual, to produce an ensemble forecasts for the original air passenger traffic series. The proposed modeling framework is called as EEMD-Slope-SVMs.

To examine the forecast performance of the proposed EEMD-Slope-SVMs, monthly air passenger traffic data of six selected airlines in UK and USA are used in the experiment to see the forecast accuracy measured by RMSE, MAPE, GMRAE, and DS compared with EEMDSVMs (not restraining the end effect), single SVMs (straightforward application of SVMs), Holt-Winters, and ARIMA.

The rest of this paper is organized as follows. Section 2 describes the related methodologies along with the proposed EEMD-based SVMs learning approach in details. Real world data series used for verification and illustration of the proposed approach are described in Section 3. Section 4 illustrates the experimental design on data preprocessing, accuracy measure, software, and parameters selection in details. Results analysis and conclusions are given in Section 5.

\section{Methodologies}

\subsection{Ensemble Empirical Mode Decomposition (EEMD)}

Ensemble empirical mode decomposition as proposed by Wu and Huang [15] is a substantial improvement over the original empirical mode decomposition (EMD) method because it avoids the problem of mode mixing. The underlying idea of EEMD is based on the understanding that the use of noise can be helpful in data analysis. Adding noise to data helps to detect the weak signals with hidden modes and to delineate the underlying processes.

EEMD is an empirical, intuitive, direct, and self-adaptive time series decomposition technique. It is suitable for decomposing nonlinear, and nonstationary time series. It decomposes original data series into intrinsic mode functions (IMFs) on the basis of local characteristic scale, the distance between two successive local extrema in EEMD. IMF must satisfy the following two requirements. (1) In the whole data series, the number of extrema (sum of maxima and minima) and the number of zero crossings must either be equal or differ at most by one. (2) At any point, the mean value of the envelopes defined by local maxima and minima must be zero.

Based on this definition, IMFs can be extracted from the data series according to the following sifting procedure:

(1) identify all the local extrema, including local maxima and local minima;

(2) connect the local maxima by a cubic spline to define the upper envelope, $X_{\mathrm{up}}(t)$, and the local minima by a second cubic spline to define the lower envelope, $X_{\text {low }}(t)$;

(3) compute the point-by-point local envelope mean $m(t)$ from the upper and lower envelopes as $m(t)=\left(X_{\text {up }}(t)+X_{\text {low }}(t)\right) / 2$;

(4) obtain the component $h$ by taking the difference between the data and the local envelope mean, $h=X(t)-m(t)$;

(5) treat $h$ as a data series and repeat step (1) to step (4) as many times as required until the envelopes are symmetric with respect to zero mean under certain criteria; 
(6) the final $h$ is designated as an IMF component $c$;

(7) obtain the residue $r$ as $r=X(t)-c$;

(8) then treat $r$ as a new data series and repeat step 1 to step 7 until the final residue becomes a monotonic function from which no more IMFs can be extracted.

Generally, the process from step (1) to step (6) is called the IMF extraction process, and the process from step (1) to step (8) is called the whole sifting process. After finishing the whole sifting process, the data series $X(t)$ can be decomposed into IMFs and a residue, that is,

$$
x(t)=\sum_{i=1}^{n} c_{j}+r_{n}
$$

where $n$ is the number of IMFs, $c_{j}(j=1,2, \ldots, n)$ are the IMFs, and $r_{n}$ is the residue, which represents the overall trend of the data series $X(t)$.

The sifting process described above is the core of the EEMD method. In implementation, some algorithm issues arise, such as the stopping criteria for IMF extraction and for the whole sifting process; a recent detailed discussion of these issues can be found in references $[16,17]$. In this study, the number of sifting passes for IMF extraction is fixed at 10, and the whole sifting process stops after $\log _{2} N$ IMFs have been extracted, where $N$ is the length of the data series.

The principal concept of the EEMD approach is as follows: the added white noise presents a uniform reference frame in the time-frequency and time-scale domains for signals of comparable scales to collate into one IMF and then cancel themselves out by ensemble averaging after serving their purpose. Thus, the problem of mode mixing in the original EMD method can be limited significantly.

For a given data series $x(t)$, the EEMD procedure can be described as follows.

(1) Generate series with added white noise, $x_{i}(t)=x(t)+w_{i}(t)$.

(2) Decompose the $x_{i}(t)$ by the sifting process described above and obtain the IMF components, $\sum_{j=1}^{n} c_{i j}+r_{i n}$.

(3) Repeat step (1) and step (2) $m$ times with a different white noise series each time; then obtain a set of IMF components, $\sum_{i=1}^{m}\left(\sum_{j=1}^{n} c_{i j}+r_{i n}\right)$, where $m$ is the ensemble number.

(4) Obtain the (ensemble) means of the corresponding IMFs of the decompositions as the final result, that is, the $j$ th ensemble IMF, $\overline{c_{j}}=(1 / m) \sum_{i=1}^{m} c_{i j}$, and the ensemble residue, $\overline{r_{n}}=(1 / m) \sum_{i=1}^{m} r_{i n}$.

In reality, the number of ensemble members is often set to 100; the standard deviation of the added white noise is set to 0.1 or 0.2 .

As discussed in [18], however, the two ends of the time series will disperse while the series is decomposed by EEMD and this disperse, termed as end effect, would "empoison" in by the whole time series gradually which makes the results to get distortion. To be more specific, end effect occurs during the shifting process, when the end points cannot be identified as the extrema, appealing specific measure to be taken to deal with it.

Recently, a large number of studies have developed end condition methods for restraining the end effect [14, 18-20]. Most of the proposed end condition methods 
are applied to "add" the extrema when end effect occurs, facilitating the construction of upper and lower envelopes during the shifting process of EEMD.

\subsection{EEMD with Slope-Based Method}

Just as mentioned in Section 2.1, the sifting process is the key part of the EEMD method and end effect occurs during the shifting process, when the end points cannot be identified as the extrema, appealing end condition methods to be incorporated into the shifting process. Thus, the slope-based method proposed by Dätig and Schlurmann [14] is adopted for dealing with end effect in the current study. The improved shifting process with slope-based method is depicted in Figure 1.

\subsection{Proposed EEMD-Based SVM Modeling Framework}

Suppose $X(t)(t=1,2, \ldots, n)$ is a time series for in sample training. Building upon the previous techniques and methods, an EEMD-based SVM ensemble learning process is formulated as the following procedures.

(1) The original time series $X(t)$ is decomposed into $m$ intrinsic mode functions components, $c_{j}(t), j=1,2, \ldots, m$ and the residual component $r_{j}(t)$ using EEMD with slope-based method presented in Section 2.2.

(2) Employ SVMs to model each IMF components and the residual component using a rolling origin and a rolling window training strategy to get the model specifications of each components, respectively.

(3) For the purpose of seeking the ensemble function, an SVM model is established to model the relationship between the actual value and the forecast values of all extracted components in the same time points. For instance, $F_{i j}(i=1,2, \ldots, n ; j=$ $1,2, \ldots, m)$ denotes the forecast of $j$ th component at $i$ th time; the ensemble SVM is to model the relationship between $X_{i}$ and $\left(F_{i 1}, F_{i 2}, \ldots, F_{i m}\right)$.

We name the proposed approach above as EEMD (decomposition)-Slope-based method (restraining end effect)-SVMs (forecasting) (abbreviated to EEMD-Slope-SVMs). Following the same naming rule, EEMD-SVMs refers to the model without any end condition methods.

Figure 2 illustrates the framework of the proposed approach.

\section{The Data}

In this study, air passenger traffic series from six airlines in USA and UK are chosen as experimental samples. The data of UK are freely obtainable from CAA (http://www.caa.co.uk/) and the data of USA are freely obtainable from the Bureau of Transportation Statistics, US Department of Transportation (http://www.bts.gov/). The main reason of selecting these two states is that the air industries in these two states started early and have a rapid development. And the reason to choose the six airlines is that these airlines are famous in these two countries and they represent the development trend of air industry in these two countries. 


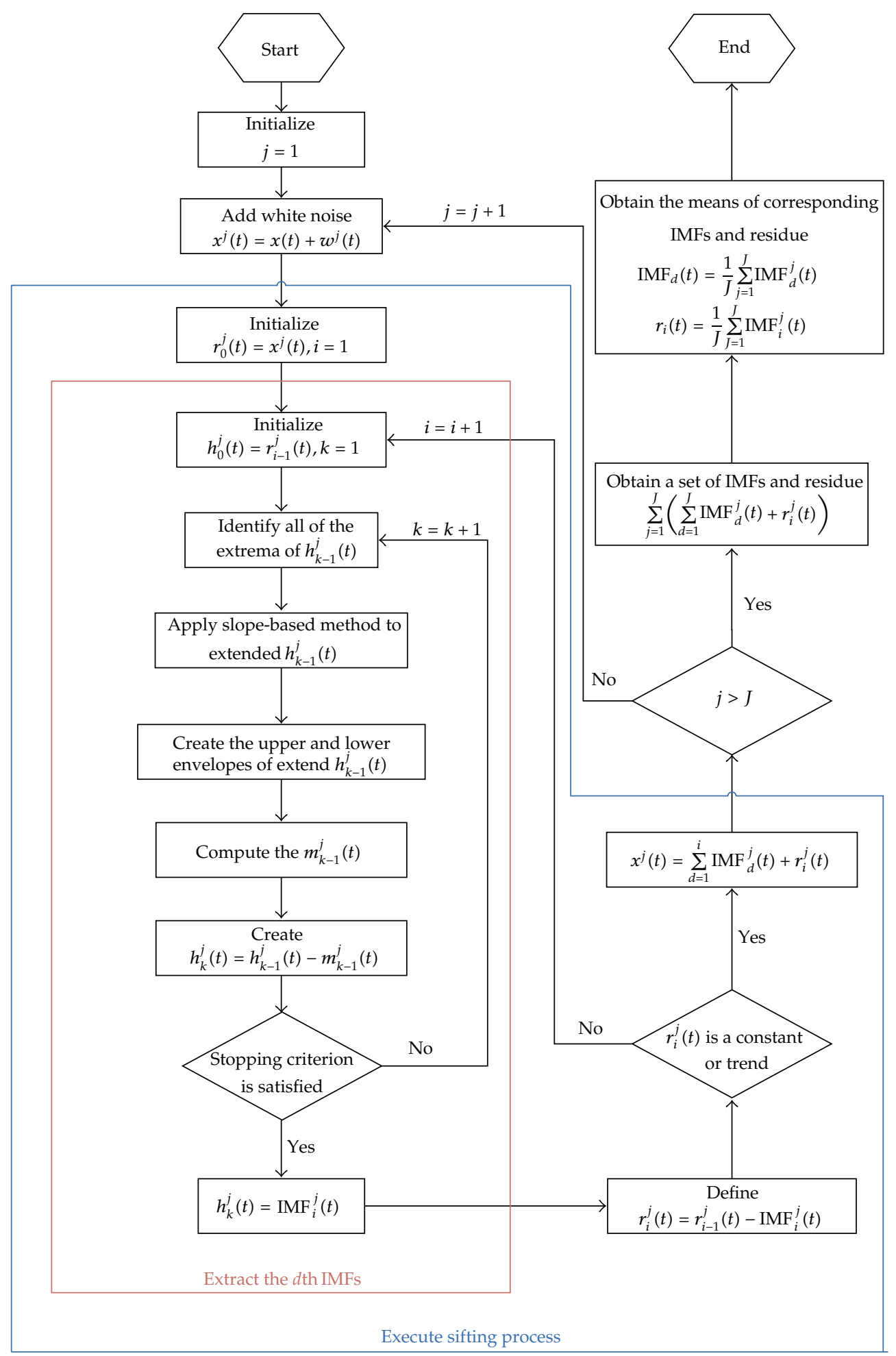

Figure 1: The EEMD with slope-based method. 


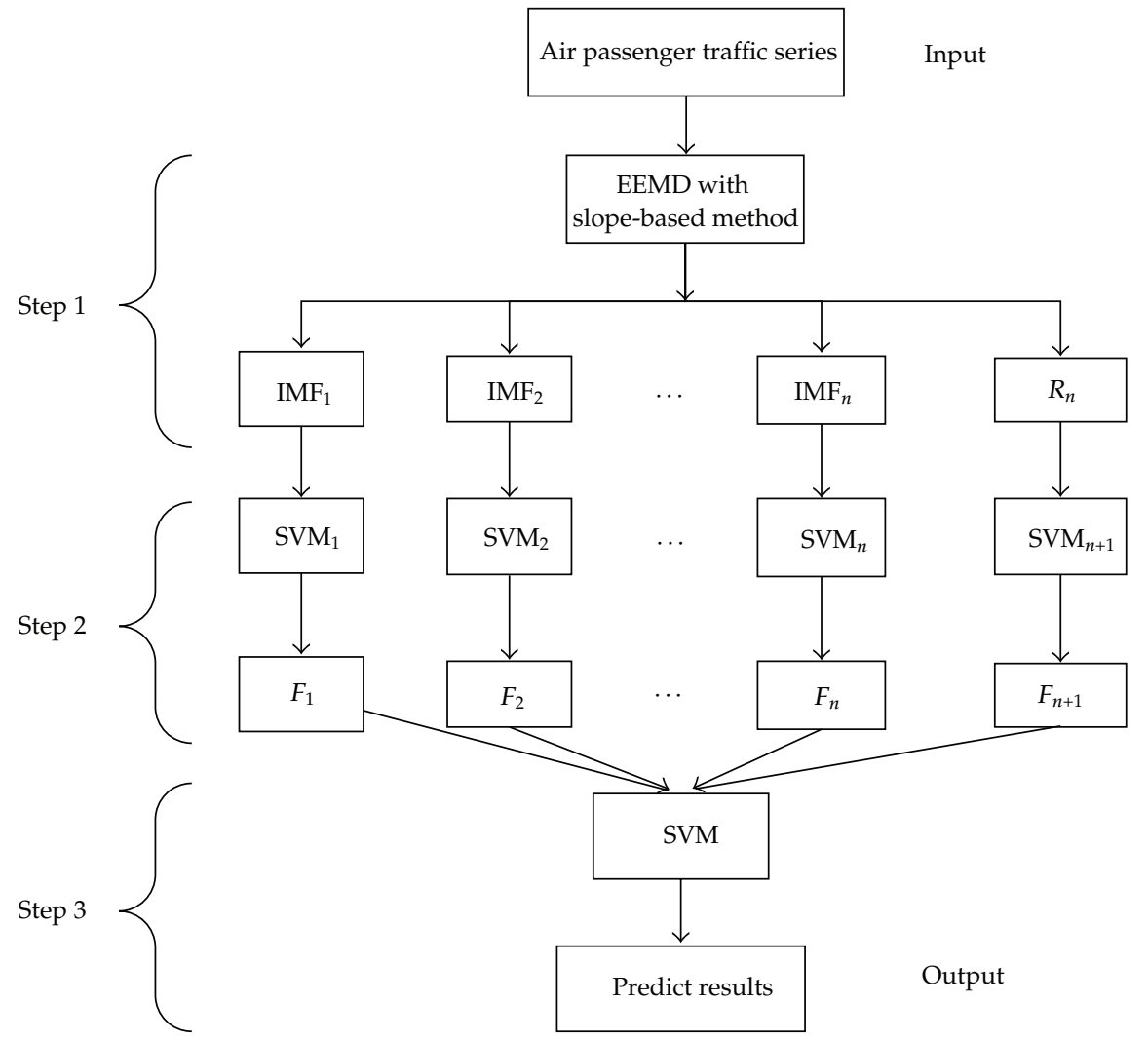

Figure 2: The framework of proposed EEMD-based SVM learning approach.

For United Air, American Airlines, and Delta Airlines, the sampling data covers the period from January 1990 to March 2008, with a total 219 observations. The data from January 1990 to March 2006 is used for the training set (195 observations), and the remainder is used as the testing set. For Southwest Airlines, we take a little longer monthly data from January 1990 to June 2008, with a total of 222 observations. The first 198 data, from January 1990 to June 2006, are used as the training set, and the remainder is used as the testing set.

For each of the two UK airlines, easyJet Airline and Virgin Atlantic Airways, the sampling data period covers from January 1998 to September 2007, with a total of 117 observations. We use the data from January 1998 to September 2005 as the training set (93 observations) and the remainder as the testing set.

\section{Experiment Design}

\subsection{Data Preprocessing}

Normalization is a standard requirement for time series modeling and forecasting. Thus, the air passenger traffic series were firstly preprocessed by adopting liner transference to adjust the original data set scaled into the range of $[0,1]$.

As previously stated, most of the air passenger traffic series considered exhibit a strong seasonal component or trend pattern. After the linear transference, deseasonalizing 
and detrending were performed. We conducted deseasonalizing by means of the revised multiplicative seasonal decomposition presented in [21]. Detrending was performed by fitting a polynomial time trend and then subtracting the estimated trend from the series when trends were detected by the Mann-Kendall test [22].

\subsection{Accuracy Measure}

The prediction performance is evaluated using the following statistical metrics, namely, the root mean squared error (RMSE), mean absolute percentage error (MAPE), and geometric mean relative absolute error (GMRAE). Let $Y_{t}$ denote the observation at time $t$ and let $F_{t}$ denote the forecast of $Y_{t}$. Then define the forecast error as $e_{t}=Y_{t}-F_{t}$ and the percentage error as $p_{t}=100 e_{t} / Y_{t}$. Let $r_{t}=e_{t} / e_{t}{ }^{*}$ denote the relative error, where $e_{t}{ }^{*}$ is the forecast error obtained from the base method. Usually, the base method is the "naïve method" where $F_{t}$ is equal to the last observation. These statistic metrics are calculated as follows:

$$
\begin{aligned}
\operatorname{RMSE} & =\sqrt{\operatorname{mean}\left(e_{t}^{2}\right),} \\
\mathrm{MAPE} & =\operatorname{mean}\left(\left|p_{t}\right|\right), \\
\mathrm{GMRAE} & =\operatorname{gmean}\left(\left|r_{t}\right|\right) .
\end{aligned}
$$

Besides accuracy, we also take the directional predictions to improve decision. The ability to predict movement direction can be measured by a directional statistic $\left(D_{\text {stat }}\right)$, which can be expressed as

$$
D_{\text {stat }}=\frac{1}{N} \sum_{t=1}^{N} a_{t} \times 100 \%, \quad a_{t}= \begin{cases}1, & \left(Y_{t}-F_{t-1}\right)\left(F_{t}-F_{t-1}\right) \geq 0 \\ 0, & \text { otherwise }\end{cases}
$$

\subsection{Benchmarking Forecasting Methods}

Holt-Winters and ARIMA are used as the benchmarking forecasting methods to justify the performance of the proposed approach in the present study. For the reason of length limit, details of Holt-Winters and ARIMA are omitted. It is worth noting that these two models utilize the original time series for forecast and do not use the decomposed ones.

\subsection{Wilcoxon's Signed-Rank Test}

In this study, a nonparametric Wilcoxon's signed-rank test [23] is performed to determine if there is a significant difference between the two approaches based on the prediction error of the testing data sets. This test performs a two-sample rank test for the difference between two population medians. Since the population distributions of the performance measures are unknown, a nonparametric test is suggested for the performance comparison of the two models [24]. 


\subsection{Software}

In this study, we employ LibSVMs (version 2.86.) [25] for SVM modeling. Holt-Winters and ARIMA models are implemented using a forecast package in $R$ (version 1.13) [26]. EEMD is implemented using the program provided by Wu and Huang [15] (http://rcada.ncu .edu.tw/). Based on LibSVMs and EEMD program, we develop our proposed EEMD-SVM programs in Matlab, which is available upon request.

\subsection{Parameters Selection}

The most important thing in SVM training is the kernel function parameters tuning. In this study, we chose the RBF as the kernel function. For the size of all data series is not very long, the efficiency of the training SVM model is not the key point; we use grid search on tuning the RBF parameters: $C$ and $\gamma$.

The parameters selection is carried out during the training for Holt-Winters and ARIMA using the embedded autofitting function in a forecast package in $R$ [26].

\section{Results and Conclusions}

The forecasting performances on testing sets of all the examined models (EEMD-SlopeSVMs, EEMD-SVMs, individual SVMs, Holt-Winters, and ARIMA) in terms of RMSE, MAPE, GMRAE, and DS for the six airlines monthly air passenger traffic data series are shown in Table 1. Additionally, the Wilcoxon's signed-rank tests for EEMD-SVMs against the three individual models and EEMD-Slope-SVMs against the counterparts are shown in Tables 2 and 3 , respectively.

Generally speaking, the goals of the experimental study are twofold. One is to examine how significant improvement can be achieved by using the hybrid decomposition and ensemble framework. The other is to examine if restraining the end effect by incorporating slope-based method into the EEMD-based SVM modeling framework can improve the performance further.

Focusing on the first goal, this is to say, by comparing the forecasting performances between hybrid EEMD-based models and individual models, two conclusions can be drawn. Note that the most significant improvement is witnessed while comparing the EEMD-SVMs and individual SVMs. For example, the average MAPE of EEMD-SVMs on six data series is 2.406, while individual SVMs is 2.913. And so do the GMRAE and DS. It should be noted that RMSE is an absolute measure and averaging it makes no sense for comparison. These results indicate that EEMD can facilitate the modeling for forecasting by decomposing the original complex data series into several simple time series. Furthermore, the individual SVMs outperform than other individual methodologies, indicating it as a promising alternative for individual modeling tasks. The Wilcoxon's signed-rank tests for EEMD-SVMs against the three individual models also statistically support the promising performance of the EEMD-SVMs with $\alpha=0.05$ significance level. Note that this study focuses on the potential improvement of the decomposition-ensemble framework with EEMD and comparative details among all the three individual models (SVMs, Holt-winter, and ARIMA) are not shown and discussed.

As for the comparison between EEMD-Slope-SVMs and EEMD-SVMs to examine the corresponding improvement of restraining the end effect, the experimental results indicate 
Table 1: Forecasting performances of all models across all the data series.

\begin{tabular}{|c|c|c|c|c|c|c|}
\hline & & EEMD-Slope-SVMs & EEMD-SVMs & SVMs & Holt-Winters & ARIMA \\
\hline \multirow{4}{*}{ American } & MAPE & 1.501 & 1.8332 & 2.096 & 5.7316 & 3.1582 \\
\hline & RMSE & 127192 & 141840 & 161874 & 449709 & 251254 \\
\hline & GMRAE & 0.2165 & 0.2973 & 0.3351 & 0.8012 & 0.5268 \\
\hline & DS & 1 & 1 & 1 & 0.6087 & 0.739 \\
\hline \multirow{4}{*}{ Delta } & MAPE & 3.815 & 4.552 & 5.4158 & 7.4413 & 6.9646 \\
\hline & RMSE & 276491 & 308793 & 331704 & 403902 & 408285 \\
\hline & GMRAE & 0.5018 & 0.5216 & 0.7141 & 0.8618 & 1.1068 \\
\hline & DS & 0.8261 & 0.7391 & 0.7261 & 0.6957 & 0.6522 \\
\hline \multirow{4}{*}{ Southwest } & MAPE & 1.1369 & 1.2937 & 1.2956 & 5.8553 & 6.0091 \\
\hline & RMSE & 150190 & 150777 & 151706 & 696873 & 632114 \\
\hline & GMRAE & 0.208 & 0.251 & 0.2643 & 0.8126 & 1.1055 \\
\hline & DS & 0.936 & 0.927 & 0.913 & 0.695 & 0.522 \\
\hline \multirow{4}{*}{ United } & MAPE & 1.3901 & 1.9475 & 2.1505 & 6.5151 & 4.0872 \\
\hline & RMSE & 96132 & 106727 & 120961 & 393550 & 251327 \\
\hline & GMRAE & 0.2091 & 0.2963 & 0.3811 & 1.1877 & 0.801 \\
\hline & DS & 0.9774 & 0.9565 & 0.913 & 0.608 & 0.913 \\
\hline \multirow{4}{*}{ easyJet } & MAPE & 2.017 & 2.9321 & 3.8019 & 5.5213 & 5.6763 \\
\hline & RMSE & 74361 & 81931 & 102859 & 175269 & 175404 \\
\hline & GMRAE & 0.4401 & 0.5183 & 0.6872 & 0.526 & 0.525 \\
\hline & DS & 0.9107 & 0.8696 & 0.78261 & 0.6087 & 0.6075 \\
\hline \multirow{4}{*}{ Virgin } & MAPE & 1.4078 & 1.8766 & 2.7149 & 4.1953 & 3.659 \\
\hline & RMSE & 10191 & 11150 & 14150 & 22395 & 18455 \\
\hline & GMRAE & 0.4109 & 0.4655 & 0.5412 & 0.6303 & 0.6515 \\
\hline & DS & 0.8991 & 0.8696 & 0.7826 & 0.6522 & 0.7391 \\
\hline
\end{tabular}

Table 2: Wilcoxon's signed-rank test for EEMD-SVMs against the three individual models.

\begin{tabular}{lccc}
\hline Metrics & EEMD- SVMs versus SVMs & EEMD- SVMs versus Holt-Winter & EEMD- SVMs versus ARIMA \\
\hline MAPE & $0.0313^{*}$ & $0.0313^{*}$ & $0.0313^{*}$ \\
RMSE & $0.0313^{*}$ & $0.0313^{*}$ & $0.0313^{*}$ \\
GMRAE & $0.0313^{*}$ & $0.0313^{*}$ & $0.0313^{*}$ \\
DS & 0.0625 & $0.0313^{*}$ & $0.0313^{*}$ \\
\hline
\end{tabular}

*Achieving 5\% significance levels, respectively (2-tailed).

Table 3: Wilcoxon's signed-rank test for EEMD-Slope- SVMs against the counterparts.

\begin{tabular}{lcccc}
\hline Metrics & $\begin{array}{c}\text { EEMD-Slope-SVMs } \\
\text { versus EEMD-SVMs }\end{array}$ & $\begin{array}{c}\text { EEMD-Slope- } \\
\text { SVMs versus SVMs }\end{array}$ & $\begin{array}{c}\text { EEMD-Slope-SVMs } \\
\text { versus Holt-Winter }\end{array}$ & $\begin{array}{c}\text { EEMD-Slope-SVMs } \\
\text { versus ARIMA }\end{array}$ \\
\hline MAPE & $0.0313^{*}$ & $0.0313^{*}$ & $0.0313^{*}$ & $0.0313^{*}$ \\
RMSE & $0.0313^{*}$ & $0.0313^{*}$ & $0.0313^{*}$ & $0.0313^{*}$ \\
GMRAE & $0.0313^{*}$ & $0.0313^{*}$ & $0.0313^{*}$ & $0.0313^{*}$ \\
DS & 0.0625 & 0.0625 & $0.0313^{*}$ & $0.0313^{*}$ \\
\hline
\end{tabular}

*Achieving 5\% significance levels, respectively (2-tailed). 
that the proposed EEMD-Slope-SVMs outperform the EEMD-SVMs and the rest three individual models in all cases across all the four metrics. The Wilcoxon's signed-rank tests for EEMD-Slope-SVMs against the counterparts statistically support the promising performance of EEMD-Slope-SVM approach with $\alpha=0.05$ significance level.

Due to the complex and dynamic pattern with nonlinearity and nonstationarity as well as implicit seasonality, air passenger traffic forecasting still remains as one the most challenging task in the field of air transportation management. This study steps on the way to establish hybrid learning framework for time series modeling and forecasting and contributes to examine the EEMD-based SVM modeling framework with slope-based method through extensive experiments.

Generally speaking, in terms of the experimental results presented in this study, we can draw the following conclusions. (1) EEMD-based SVM modeling frameworks achieve better than the individual models. (2) The proposed EEMD-Slope-SVM modeling framework outperforms EEMD-SVMs and the rest three individual models achieve the best performance. This indicates that restraining the end effect occurring during the shifting process of EEMD can be helpful to improve the prediction performance further.

This study also has limitation in the selection of the methods to restrain the end effect. There are several other methods in the literature and only slope-based method is examined in this study. More extensive studies on the other methods should be conducted and it remains as a future research topic.

\section{Acknowledgments}

This work was supported by the Natural Science Foundation of China under Project No. 70771042, the Fundamental Research Funds for the Central Universities (2012QN208-HUST), and a Grant from the Modern Information Management Research Center at Huazhong University of Science and Technology.

\section{References}

[1] S. Y. Abed, A. O. Ba-Fail, and S. M. Jasimuddin, "An econometric analysis of international air travel demand in Saudi Arabia," Journal of Air Transport Management, vol. 7, no. 3, pp. 143-148, 2001.

[2] H. Grubb and A. Mason, "Long lead-time forecasting of UK air passengers by Holt-Winters methods with damped trend," International Journal of Forecasting, vol. 17, no. 1, pp. 71-82, 2001.

[3] DETR, Air Traffic Forecast for the United Kingdom, in Department of the Environment, Transport and the Regions, 2000.

[4] DFT, “The Future of Air Transport," Department for Transport, 2003.

[5] DFT, Passenger Forecasts: Additional Analysis, Department for Transport, 2003.

[6] DFT, UK Air Passenger Demand and $\mathrm{CO}_{2}$ Forecasts, Department for Transport, 2007.

[7] W. E. O'Connor, An Introduction to Airline Economics, Praeger Publishers, 2001.

[8] J. D. Bermúdez, J. V. Segura, and E. Vercher, "Holt-Winters forecasting: an alternative formulation applied to UK air passenger data," Journal of Applied Statistics, vol. 34, no. 9-10, pp. 1075-1090, 2007.

[9] K. Nam and T. Schaefer, "Forecasting international airline passenger traffic using neural networks," Logistics and Transportation Review, vol. 31, no. 3, 1995.

[10] N. Kyungdoo, Y. Junsub, and R. P. Victor, "Predicting airline passenger volume," The Journal of Business Forecasting Methods \& Systems, vol. 16, no. 1, pp. 14-16, 1997.

[11] L. Yu, S. Wang, and K. K. Lai, "Forecasting crude oil price with an EMD-based neural network ensemble learning paradigm," Energy Economics, vol. 30, no. 5, pp. 2623-2635, 2008.

[12] C. F. Chen, M. C. Lai, and C. C. Yeh, "Forecasting tourism demand based on empirical mode decomposition and neural network," Knowledge-Based Systems, vol. 26, pp. 281-287, 2011. 
[13] Y. Wei and M. C. Chen, "Forecasting the short-term metro passenger flow with empirical mode decomposition and neural networks," Transportation Research C, vol. 21, no. 1, pp. 148-162, 2012.

[14] M. Dätig and T. Schlurmann, "Performance and limitations of the Hilbert-Huang transformation (HHT) with an application to irregular water waves," Ocean Engineering, vol. 31, no. 14-15, pp. 17831834, 2004.

[15] Z. Wu and N. E. Huang, "Ensemble empirical mode decomposition: a noise-assisted data analysis method," Advances in Adaptive Data Analysis, vol. 1, no. 1, pp. 1-41, 2009.

[16] N. E. Huang, M.-L. C. Wu, S. R. Long et al., "A confidence limit for the empirical mode decomposition and Hilbert spectral analysis," The Royal Society of London. Proceedings A, vol. 459, no. 2037, pp. 23172345, 2003.

[17] S. S. P. Shen, T. Shu, N. E. Huang et al., "HHT analysis of the nonlinear and non-stationary annual cycle of daily surface air temperature data," in Hilbert-Huang Transform and its Applications, vol. 5, pp. 187-209, 2005.

[18] J. Cheng, D. Yu, and Y. Yang, "Application of support vector regression machines to the processing of end effects of Hilbert-Huang transform," Mechanical Systems and Signal Processing, vol. 21, no. 3, pp. 1197-1211, 2007.

[19] G. Rilling, P. Flandrin, and P. Gonçalvés, On empirical mode decomposition and its algorithms, 2003.

[20] F. Wu and L. Qu, "An improved method for restraining the end effect in empirical mode decomposition and its applications to the fault diagnosis of large rotating machinery," Journal of Sound and Vibration, vol. 314, no. 3, pp. 586-602, 2008.

[21] R. R. Andrawis, A. F. Atiya, and H. El-Shishiny, "Forecast combinations of computational intelligence and linearmodels for the NN5 time series forecasting competition," International Journal of Forecasting, vol. 27, no. 3, pp. 672-688, 2011.

[22] B. Onoz and M. Bayazit, "The power of statistical tests for trend detection," Turkish Journal of Engineering and Environmental Sciences, vol. 27, no. 4, pp. 247-251, 2003.

[23] X. Francis and S. Roberto, "Comparing Predictive Accuracy," Journal of Business E Economic Statistics, vol. 13, no. 3, 1995.

[24] W. J. Conover, Practical Nonparametric Statistics, vol. 3, John Wiley \& Sons, New York, NY, USA, 1980.

[25] C. C. Chang and C. J. Lin, "LIBSVM: a library for support vector machines," ACM Transactions on Intelligent Systems and Technology (TIST), vol. 2, no. 3, p. 27, 2011.

[26] Y. Khandakar and R. J. Hyndman, "Automatic time series forecasting: the forecast Package for R," Journal of Statistical Software, vol. 27, no. i03, 2008. 


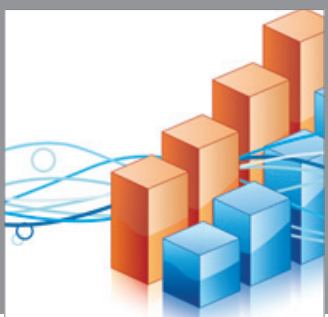

Advances in

Operations Research

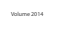

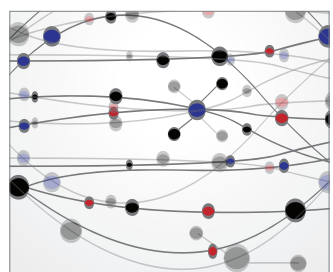

\section{The Scientific} World Journal
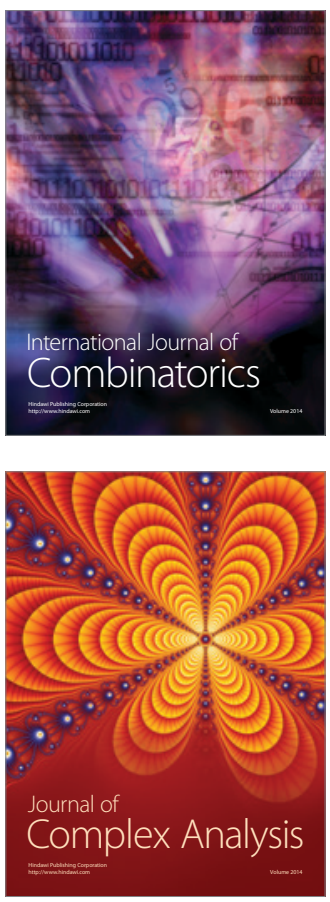

International Journal of

Mathematics and

Mathematical

Sciences
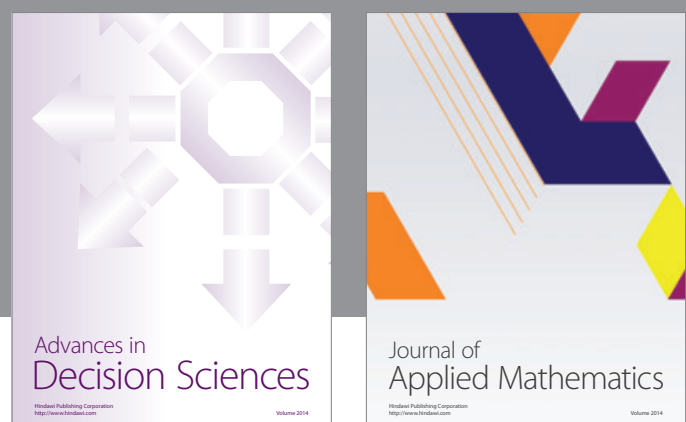

Journal of

Applied Mathematics
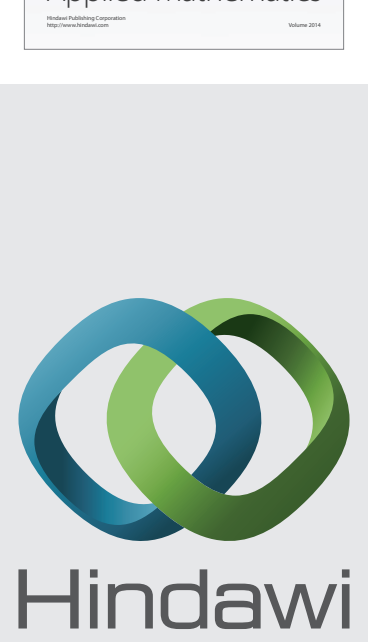

Submit your manuscripts at http://www.hindawi.com
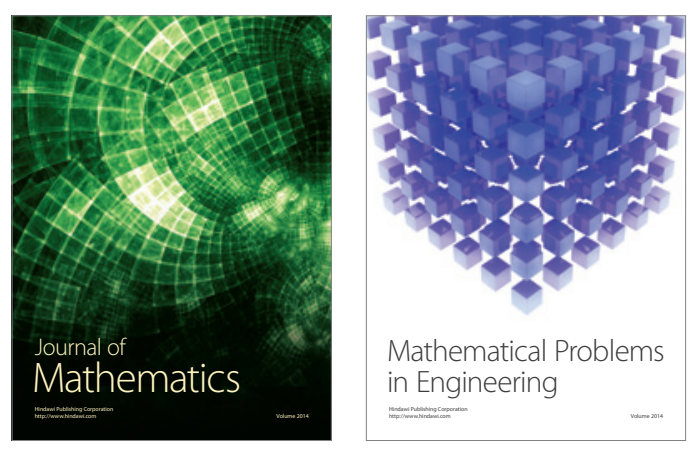

Mathematical Problems in Engineering
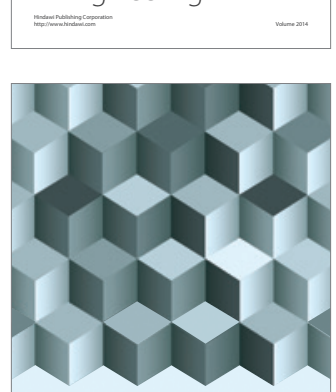

Journal of

Function Spaces
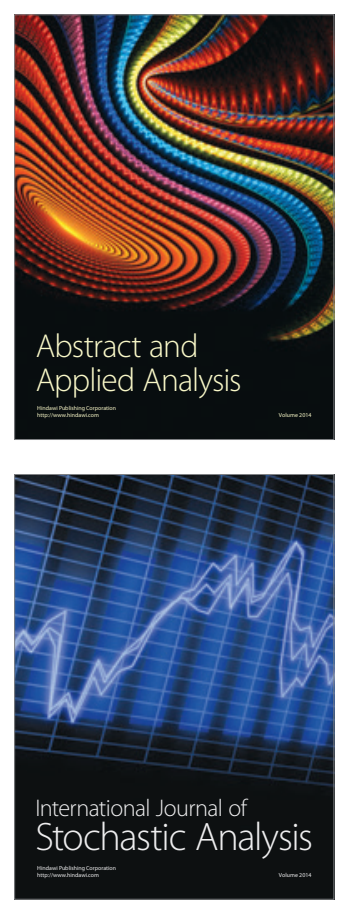

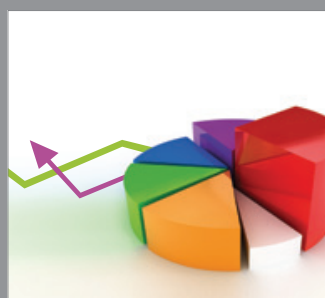

ournal of

Probability and Statistics

Promensencen
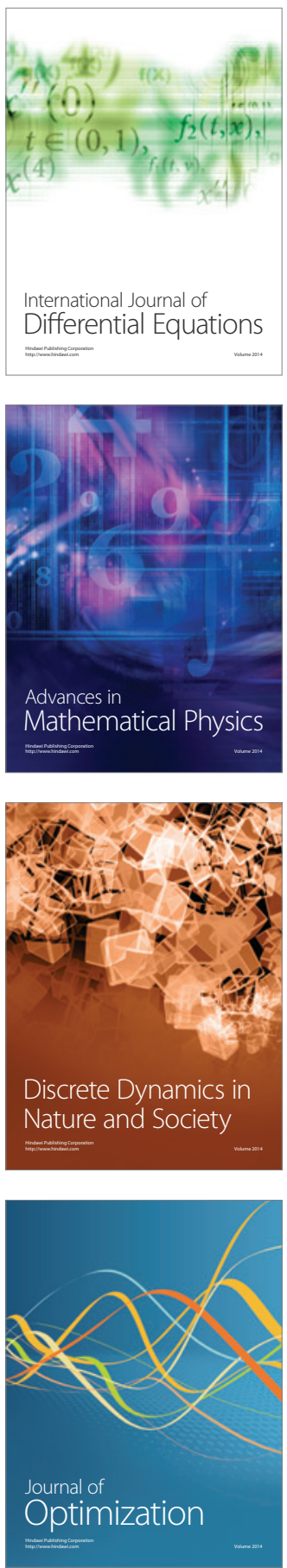